\title{
Lysosomal Abnormalities in Hadacidin-treated Dictyostelium discoideum Amoebae
}

\author{
By EDMUND V. CREAN, J. PETER LAGERSTEDT AND \\ EDWARD F. ROSSOMANDO* \\ Department of Oral Biology, School of Dental Medicine, University of Connecticut \\ Health Center, Farmington, Connecticut 06032, U.S.A.
}

(Received 15 July 1980)

When Dictyostelium discoideum amoebae were harvested from nutrient medium and suspended in a starvation buffer to initiate development, approximately $30 \%$ of the total cellular $\beta$ - $N$-acetylglucosaminidase activity was secreted into the extracellular fluid within $4 \mathrm{~h}$. During this same period, only $10 \%$ of the total cellular acid phosphatase and acid protease activities were secreted. When the cells were pretreated overnight with $5 \mathrm{mg}$ sodium hadacidin $\mathrm{ml}^{-1}$ and then suspended in starvation buffer, $60 \%$ of the glucosaminidase and $30 \%$ of the acid phosphatase activities were secreted, while the level of acid protease secretion remained at $10 \%$. The secretory behaviour of hadacidin-treated cells was, however, identical to that of untreated cells when $0.1 \mathrm{M}$-sucrose was added to the starvation buffer to enhance lysosomal enzyme secretion.

Treatment with hadacidin also affected the intracellular content of these enzyme activities. After $16 \mathrm{~h}$ exposure to $5 \mathrm{mg}$ hadacidin $\mathrm{ml}^{-1}$, the cellular levels of glucosaminidase and acid protease activity were decreased by $50 \%$ and $30 \%$, respectively, while acid phosphatase activity remained unchanged. All of the changes observed upon hadacidin treatment were time dependent and were not evident if the cells were exposed to the drug for only $4 \mathrm{~h}$. These results suggest that hadacidin treatment affects the lysosomal system of $D$. discoideum.

\section{INTRODUCTION}

In a previous study it was reported that when Dictyostelium discoideum amoebae are harvested from growth medium and suspended in a nutrient-free phosphate buffer (starvation buffer) the cells release 20 to $30 \%$ of their total cellular $\beta$-N-acetylglucosaminidase activity (Crean \& Rossomando, 1979) as well as an acid protease activity (Rossomando et al., $1978 b$ ). The combination of these results suggested that a variety of lysosomal enzyme activities are secreted at the onset of starvation. In addition, it was observed that the addition of sucrose (or other sugars) to the starvation buffer enhances the secretion of lysosomal glycosidase activities to the extent that over $90 \%$ of the total cellular activities are secreted (Crean \& Rossomando, 1979).

To study the secretion of these lysosomal activities further, we explored the implications of other studies (Mato et al., 1966; Angelici \& Pourtois, 1968; Fairbanks \& Kollar, 1974) which suggested to us that treating cells with hadacidin, a drug which we had previously shown to affect several cell surface properties in D. discoideum (Rossomando et al., 1978a), might alter the process of secretion of lysosomal enzyme activities. In this report, we examine the secretory properties of hadacidin-treated cells in comparison with those of untreated cells. In addition, we have studied the secretory response of hadacidin-treated cells to sucrose, again comparing this response to that of untreated cells. 


\section{METHODS}

Materials. Growth medium components were obtained from BBL. Sucrose and all inorganic chemicals were purchased from Baker Chemical. $p$-Nitrophenyl- $\beta$ - $N$-acetylglucosaminide and $p$-nitrophenyl phosphate were from Sigma. Sodium hadacidin was generously supplied by Merck, Sharp, and Dohme. Hide powder azure was obtained from Calbiochem.

Growth of organisms and hadacidin treatment. An axenic strain (Ax-3) of Dictyostelium discoideum (parent strain, NC-4 haploid) was grown in HL-5 medium as previously described (Rossomando et al., 1974). To initiate starvation, cells were harvested during exponential growth and resuspended at $1 \times 10^{7}$ to $2 \times 10^{7}$ cells $\mathrm{ml}^{-1}$ in a starvation buffer containing $17 \mathrm{mM}$-potassium phosphate (pH 6.1) and $2 \mathrm{mM}-\mathrm{MgSO}_{4}$. For hadacidin treatment, the drug was added to an exponential-phase suspension of cells in HL-5 medium to a final concentration of $5 \mathrm{mg} \mathrm{ml}^{-1}$. The suspension was shaken overnight $(16 \mathrm{~h})$ at $22^{\circ} \mathrm{C}$ and the cells were then harvested for secretion studies. These cells are referred to as hadacidin-treated cells.

Secretion studies. Secretion of lysosomal enzyme activities was measured as previously described (Crean \& Rossomando, 1979). In brief, amoebae were harvested and suspended as described above in either starvation buffer alone or starvation buffer containing $0.1 \mathrm{M}$-sucrose. At $1 \mathrm{~h}$ intervals, $1 \mathrm{ml}$ of each suspension was removed and the cells were separated from the extracellular fluid by brief centrifugation. The cell pellet was dissolved in $1 \mathrm{ml} 1 \%(\mathrm{v} / \mathrm{v})$ Triton X-100 while the extracellular fluid was mixed with $0.1 \mathrm{ml} 1 \%$ Triton X-100. These solutions were stored at $4{ }^{\circ} \mathrm{C}$ until used for enzyme assays.

Enzyme assays. $\beta$ - $N$-Acetylglucosaminidase (EC 3.2.1.30) and acid phosphatase (EC 3.1.3.2) activities were assayed as previously described (Crean \& Rossomando, 1979), using $5 \mathrm{~mm}$-p-nitrophenyl substrates in $50 \mathrm{~mm}$-acetate buffer at the appropriate $\mathrm{pH}$ values. For each activity one unit is equivalent to the production of $1 \mu \mathrm{mol}$ product $\mathrm{h}^{-1}$ at $30^{\circ} \mathrm{C}$. Acid protease activity was measured using the insoluble substrate hide powder azure as previously described (Rossomando et al., 1978 b) except that the assay mixture contained $0.06 \mathrm{M}$-sodium acetate (pH 4). One unit of activity is equivalent to a change of $0.1 A_{59 s}$ units $\mathrm{h}^{-1}$ at $30^{\circ} \mathrm{C}$. Total activity refers to the sum of secreted and intracellular activity in a cell suspension.

\section{RESULTS}

\section{Lysosomal enzyme secretion from hadacidin-treated cells}

In a previous report it was shown that $D$. discoideum amoebae secrete lysosomal enzyme activities when harvested from nutrient broth and resuspended in a starvation buffer (Crean $\&$ Rossomando, 1979). For the present study we examined the secretory behaviour of hadacidin-treated cells using three representative lysosomal enzyme activities, namely, $\beta$ - $N$-acetylglucosaminidase, acid protease and acid phosphatase activities (Weiner \& Ashworth, 1970; de Chastellier \& Ryter, 1977).

When hadacidin-treated cells were suspended in starvation buffer, they secreted 60 to $70 \%$ of their total (secreted plus intracellular) glucosaminidase activity, approximately twice the percentage secreted by untreated cells (Fig. 1a). The kinetics of secretion were similar for both untreated and hadacidin-treated cells and neither population exhibited any change in total glucosaminidase activity during the $4 \mathrm{~h}$ incubation in starvation buffer. Hadacidin treatment did not affect the secretion of acid protease activity, however. Both the untreated and hadacidin-treated cells secreted about $10 \%$ of the total cellular activity when suspended in starvation buffer (Fig. $1 b$ ). Again, no change in total acid protease activity was seen during the $4 \mathrm{~h}$ starvation. The secretion of acid phosphatase activity exhibited the highest quantitative response to hadacidin treatment. While untreated cells secreted only $10 \%$ of the total acid phosphatase activity, the hadacidin-treated cells secreted $30 \%$ (Fig. $1 \mathrm{c}$ ).

Additional studies (results not shown) revealed that these effects of hadacidin treatment were time dependent. For example, cells treated with hadacidin for only $4 \mathrm{~h}$ were no different from untreated cells in their secretory activity.

\section{Lysosomal enzyme content of hadacidin-treated cells}

As shown above, comparison of the percentage of each activity secreted in $4 \mathrm{~h}$ by untreated and hadacidin-treated cells revealed that hadacidin treatment had a different quantitative effect on the secretion of each activity. Comparison of the enzyme activities in untreated and hadacidin-treated cells at the onset of starvation also revealed differential quantitative 


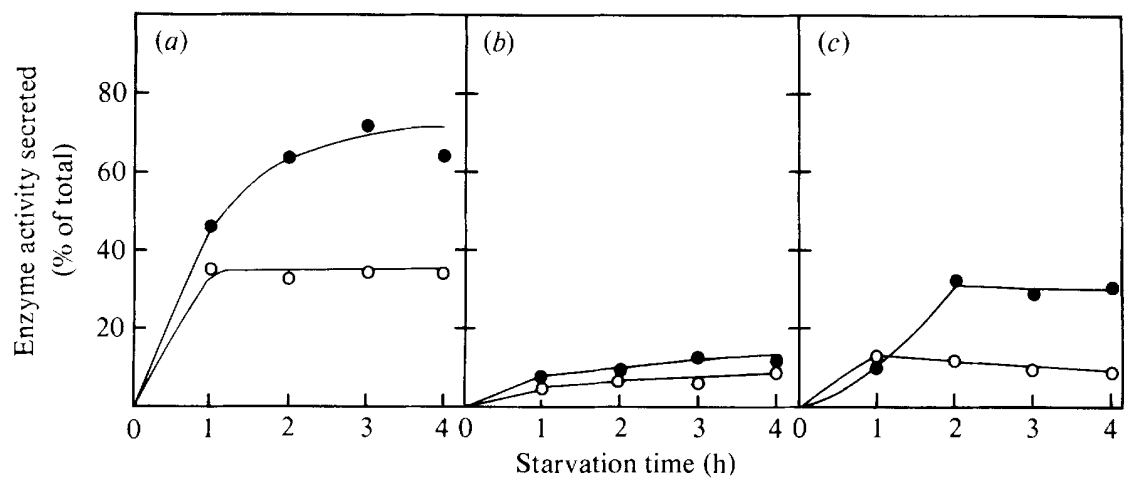

Fig. 1. Secretion of lysosomal enzyme activities in starvation buffer. Untreated (O) and hadacidintreated (O) cells were harvested and suspended in starvation buffer, and at the times indicated the percentage of total cellular activity secreted was determined for $\beta$ - $N$-acetylglucosaminidase $(a)$, acid protease $(b)$ and acid phosphatase $(c)$, as described in Methods.

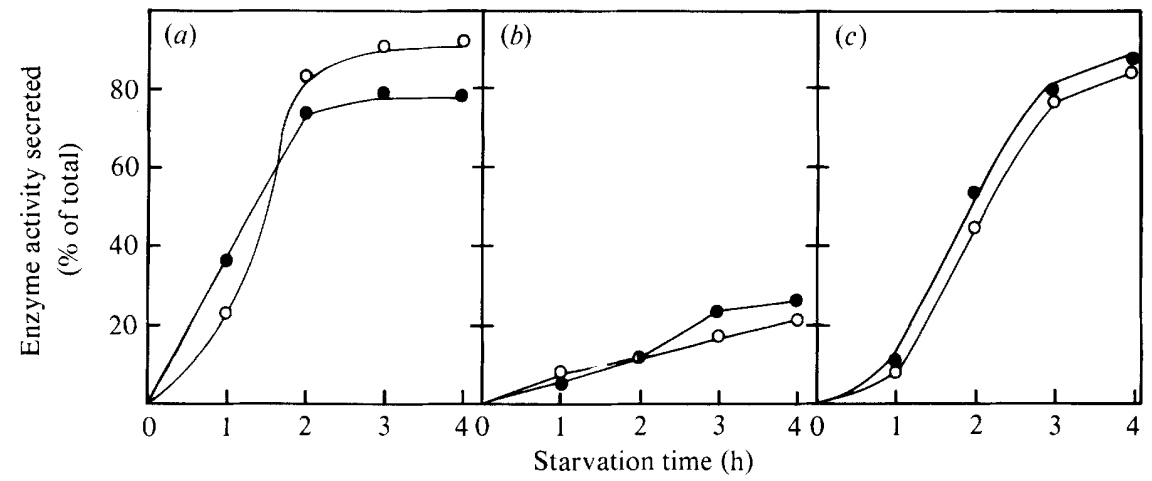

Fig. 2. Sucrose stimulation of lysosomal enzyme secretion. Untreated $(O)$ and hadacidin-treated (O) cells were harvested and suspended in starvation buffer containing $0.1 \mathrm{M}$-sucrose, and at the times indicated the percentage of total cellular activity secreted was determined for $\beta-N$-acetylglucosaminidase $(a)$, acid protease $(b)$ and acid phosphatase $(c)$.

changes (Table 1). Hadacidin-treated cells possessed $50 \%$ less total glucosaminidase activity and $30 \%$ less acid protease activity than untreated cells, while acid phosphatase activity remained essentially unchanged. Thus, the ratio of glucosaminidase to acid phosphatase to acid protease activity in untreated cells was $7: 2: 1$ in contrast to the $5: 3: 1$ ratio in hadacidin-treated cells. It should be noted that these ratios remained the same throughout the $4 \mathrm{~h}$ starvation since no changes in total activities were observed during this period. In addition, these results suggest that the higher percentages of activity secreted by hadacidin-treated cells (Fig. 1) cannot be readily explained by changes in the cellular content of these activities.

\section{Secretory response of hadacidin-treated cells to sucrose}

In a previous report it was demonstrated that addition of $0.1 \mathrm{M}$-sucrose to the starvation buffer enhanced the secretion of lysosomal enzyme activities (Crean \& Rossomando, 1979). As shown in Fig. 2, when sucrose was present in the starvation buffer, the kinetics and percentage of each activity secreted were virtually identical for untreated and hadacidintreated cells; thus the differences in the secretory profiles of the various activities in starvation buffer alone appeared to be negated by the presence of sucrose in the buffer.

There were quantitative differences between the activities in the magnitude of the response of untreated and hadacidin-treated cells to sucrose. Comparison of the data in Figs 1 and 2 
Table 1. Lysosomal enzyme activities in untreated and hadacidin-treated cells

Amoebae were harvested from HL-5 medium during the exponential growth phase (untreated) or after $16 \mathrm{~h}$ exposure to sodium hadacidin $\left(5 \mathrm{mg} \mathrm{ml}^{-1}\right)$. Cell pellets were dissolved in $1 \%$ Triton $\mathrm{X}-100$ and assayed as described in Methods.

\begin{tabular}{lcc}
\multicolumn{1}{c}{ Enzyme } & $\overbrace{\text { Untreated }}^{\text {Activity (units per } 10^{6} \text { cells) }}$ & Hadacidin-treated \\
$\beta-N$-Acetylglucosaminidase & $4 \cdot 0$ & 2.0 \\
Acid phosphatase & 1.1 & 1.2 \\
Acid protease & 0.55 & 0.38
\end{tabular}

reveals that untreated cells secreted $90 \%$ of their total glucosaminidase activity in the presence of sucrose, in contrast to the $30 \%$ secreted in starvation buffer alone - a 3-fold stimulation. The corresponding values for sucrose stimulation of acid phosphatase and acid protease secretion by untreated cells were 8 -fold and 2 -fold, respectively. For hadacidintreated cells, the sucrose stimulation of glucosaminidase, phosphatase and protease secretion was $1 \cdot 2$-fold, $2 \cdot 8$-fold and $1 \cdot 3$-fold, respectively, in comparison with secretion in starvation buffer alone. These results suggest that there is an upper limit to the percentage of each activity which can be secreted by either untreated or hadacidin-treated cells, and that the hadacidin-treated cells suspended in starvation buffer alone are already approaching this limit.

Finally, it should be noted that hadacidin-treated cells retained their selectivity with regard to substances which stimulate lysosomal enzyme secretion. As shown above, sucrose enhanced secretion in both hadacidin-treated and untreated cells (Fig. 2). In a previous report it was demonstrated that glucose or amino acids could not stimulate secretion in untreated D. discoideum (Crean \& Rossomando, 1979), and we found that these compounds were also unable to stimulate secretion in hadacidin-treated cells (results not shown).

\section{DIS CUSSION}

The results of the present study demonstrated that hadacidin treatment induces abnormalities in the lysosomal system of $D$. discoideum. A complete understanding of the mechanism by which hadacidin affects the lysosomes of $D$. discoideum will require further investigations of the normal activities of these organelles in this organism. In untreated cells, the kinetics of release of each of the activities examined suggest that the activities may all be secreted in the same manner. However, the observation that different percentages of the total amount of each activity are secreted suggests that the cells have some means of controlling how much of each activity is secreted. For example, $D$. discoideum may possess a heterogeneous population of lysosomes and the amount of secretion of each activity could be determined by which subpopulations are allowed to secrete at the cell membrane. Alternatively, the lysosomal population may be homogeneous and the percentage of each activity secreted may be regulated by varying the intralysosomal distribution of membrane-bound versus soluble enzyme.

Whatever the mechanism controlling the amount of secretion may be, it is clear that the addition of sucrose to the starvation buffer leads to differential effects on the control mechanisms for the different enzyme activities. Thus, sucrose stimulates protease secretion only 2 -fold while stimulating glucosaminidase and phosphatase secretion 3 -fold and 8 -fold, respectively. It is also interesting to note that the various activities differ in their respective rates of secretion when stimulated by sucrose (Fig. 2). In particular, there appears to be more of a lag in sucrose stimulation of acid phosphatase secretion (Fig. $2 c$ ).

Treatment of $D$. discoideum amoebae with hadacidin also leads to differential effects on the lysosomal enzyme activities. Quantitative changes in the amounts of glucosaminidase and 
acid protease activity per cell are observed, while the acid phosphatase level remains unchanged. These changes cannot be accounted for by direct inhibition of the activities by hadacidin itself (results not shown). Upon suspension in starvation buffer, the hadacidintreated cells secrete higher percentages than untreated cells of glucosaminidase and phosphatase activity, but not of protease activity (Fig. 1). The continued viability of the hadacidin-treated cells (Rossomando et al., 1978a) and the similar responses of hadacidintreated and untreated cells to sucrose (Fig. 2) indicate that the higher percentages of activity secreted by the treated cells are not due to cell lysis. In addition, it was previously shown that hadacidin-treated cells are more osmotically stable than untreated cells (Rossomando et al., 1978a).

Preliminary experiments on the effects of hadacidin treatment on $D$. discoideum development have not shown any abnormal changes in adenylate cyclase, cyclic AMP phosphodiesterase or 5'-nucleotidase activities. When hadacidin-treated cells were suspended in starvation buffer, they became aggregation competent within the same period of time as untreated cells. Yet when the two cell populations were deposited on filter pads, the hadacidin-treated cells were found to be retarded in the earlier stages of development, although they did proceed to form morphologically normal fruiting bodies (unpublished observations). Whether this retarded development is related to the lysosomal abnormalities remains to be determined.

The combination of results presented here suggests that hadacidin treatment may represent a novel probe to facilitate more detailed studies of the function of the lysosomal system of $D$. discoideum during growth and development. In addition, the ability of hadacidin to induce lysosomal abnormalities may be related to its ability to inhibit embryonic palatal fusion (Fairbanks \& Kollar, 1974). Since lysosomal elements appear to be involved in the process of palatal fusion (Mato et al., 1966; Angelici \& Pourtois, 1968) it should prove interesting to examine the effects of hadacidin treatment on the lysosomal activities associated with this process.

This research was supported in part by a grant from the National Institutes of Health DE 04657-05.

\section{REFERENCES}

Angelici, D. \& Pourtois, M. (1968). The role of acid phosphatase in the fusion of the secondary palate. Journal of Embryology and Experimental Morphology 20, 15-23.

Crean, E. V. \& Rossomando, E. F. (1979). Effects of sugars on glycosidase secretion in Dictyostelium discoideum. Journal of General Microbiology 110, 315-322.

de Chastellier, C. \& Ryter, A. (1977). Changes of the cell surface and of the digestive apparatus of Dictyostelium discoideum during the starvation period triggering aggregation. Journal of Cell Biology 75, 218-236.

FaIrbanKs, M. B. \& Kollar, E. J. (1974). Inhibition of palatal fusion in vitro by hadacidin. Teratology 9 , $169-178$.

Mato, M., Aikawa, E. \& Katahira, M. (1966). Appearance of various types of lysosomes in the epithelium covering lateral palatine shelves during secondary palate formation. Gunma Journal of Medical Sciences 15, 46-56.
Rossomando, E. F., Steffek, A. J., Mujwid, D. K. \& AleXANDER, S. (1974). Scanning electron microscopic observations on cell surface changes during aggregation of Dictyostelium discoideum. Experimental Cell Research 85, 73-78.

Rossomando, E. F., Maldonado, B. \& Crean, E. V. $(1978 a)$. Effect of hadacidin on growth and adenylosuccinate synthetase activity of Dictyostelium discoideum. Antimicrobial Agents and Chemotherapy 14, 476-482.

Rossomando, E. F., Maldonado, B., Crean, E. V. \& Kollar, E. J. $(1978 b)$. Protease secretion during onset of development in Dictyostelium discoideum. Journal of Cell Science 30, 305-318.

Weiner, E. \& Ashworth, J. M. (1970). Isolation and characterization of lysosomal particles from myxamoebae of the cellular slime mould Dictyostelium discoideum. Biochemical Journal 118, 505-512. 
